\title{
O PROCESSO DE INCLUSÃO DE AUTISTAS NO MERCADO DE TRABALHO
}

\section{THE PROCESS OF AUTISTICS INCLUSION IN THE LABOR MARKET}

\author{
Claudio Bezerra Leopoldino \\ Universidade Federal do Ceará \\ claudio.leopoldino@ufc.br
}

Pedro Felipe da Costa Coelho

Universidade Federal do Ceará pedrofelipecc@gmail.com

Submissão: $30 / 07 / 2017$

Aprovação: 08/11/2017

\begin{abstract}
RESUMO
Indivíduos com autismo enfrentam barreiras para atuar no mercado de trabalho brasileiro em função de restrições inerentes ao autismo, como limitações na comunicação, e devido a questões estruturais. A despeito de sua representatividade na população, poucas pesquisas abordam a sua inclusão no mercado de trabalho. Diante disso, este estudo objetiva apresentar o panorama do processo inclusivo de autistas no mercado de trabalho. A partir de revisão da literatura, o presente ensaio teórico aborda as políticas públicas e o processo de inclusão de pessoas dentro do Transtorno do Espectro Autista (TEA), além das dificuldades e oportunidades decorrentes deste processo. Os resultados sugerem que os dispositivos legais não foram acompanhados de estruturas para a implantação do processo inclusivo, e deveriam estimular a preparação de autistas para a atuação profissional e sua contratação. Os entraves para inclusão resultam do preconceito dos empregadores e da falta de preparo vocacional das pessoas com autismo, além da ineficácia das cotas para deficientes e ausência de incentivos. Esses obstáculos podem ser amenizados com a exploração das potencialidades das pessoas com TEA, como a facilidade em executar funções repetitivas e memorizar detalhes. A inclusão de autistas também pode ser uma boa oportunidade para empregadores e para o Estado.
\end{abstract}

Palavras-chave: Inclusão no trabalho. Transtorno do Espectro Autista. Inclusão de Deficientes. 


\begin{abstract}
Autistic individuals face barriers to work in the Brazilian labor market due to restrictions inherent to autism, such as limitations in communication, and due to structural issues. In spite of their representativeness in the population, few studies address their inclusion in the labor market. Therefore, this study aims to present the panorama of the autistic process in the labor market. From the literature review, this theoretical essay addresses public policies and the process of inclusion of people within the Autism Spectrum Disorder (ASD), as well as the difficulties and opportunities arising from this process. The results suggest that the legal mechanisms were not accompanied by structures for the implementation of the inclusive process, and should stimulate the preparation of autistic patients for professional performance and their contracting. Obstacles to inclusion stem from employer bias and lack of vocational training for people with autism, as well as ineffectiveness of quotas for the disabled and lack of incentives. These barriers can be mitigated by exploiting the potentialities of people with ASD, such as the ease of performing repetitive functions and memorizing details. The inclusion of autistic may also be a good opportunity for employers and for the state.
\end{abstract}

Keywords: Work inclusion. Autism Spectrum Disorder. Inclusion of disabled.

\title{
1 INTRODUÇÃO
}

O processo de inclusão de pessoas com Transtorno do Espectro Autista (TEA) no mercado de trabalho apresenta dificuldades e descontinuidades. Além das restrições inerentes ao autismo, como as limitações na comunicação, na socialização, comportamentos repetitivos e problemas no aparato sensorial (HARMON, 2011; BONTEMPO, 2009; KLIN, 2006), outras barreiras são enfrentadas por esse grupo de cidadãos, pois questões estruturais e culturais apresentam potencial danoso ao acesso ao trabalho (DAVIDSON, 2014; WORLD HEALTH ORGANIZATION; THE WORLD BANK, 2011; ROBERTSON, 2009; HILLIER et al., 2007).

Parte da dificuldade no processo de inclusão de autistas no trabalho pode ser atribuída a questões não resolvidas adequadamente, relacionadas ao desenvolvimento dos indivíduos. Diagnóstico tardio, restrições de acesso à educação, negação de terapias tradicionais e alternativas e baixa renda familiar estão entre os fatores que influenciam negativamente a construção de conhecimento e autonomia dos autistas e, consequentemente, sua probabilidade de aceitação no mercado laboral (TEIXEIRA, 2016; GOMES et al., 2015; MINATEL; MATSUKURA, 2015; ROUX et al., 2013; WORLD HEALTH ORGANIZATION; THE WORLD BANK, 2011). No entanto, ainda que receba tratamento adequado, o indivíduo com TEA pode não se desenvolver a ponto de poder atuar no mercado de trabalho, devido à intensidade das manifestações do autismo (BURGESS; CIMERA, 2014).

Ainda que superem os obstáculos ao desenvolvimento de suas potencialidades, as pessoas com TEA encontram novas dificuldades na transição para a vida adulta (ROUX et al., 2013). Discriminação dos colegas de trabalho (ORSMOND et al., 2013), oferta de vagas com baixa qualidade (ROUX et al., 2013), falta de preparo vocacional (HILLIER et al., 2007) e preconceito dos potenciais empregadores (SILVA, 2013; ROBERTSON, 2009) são algumas das ocorrências frequentemente relatadas na literatura internacional. Adicionalmente, a literatura também menciona a falta de serviços voltados para os autistas após a saída do ambiente escolar, a partir da transição para a vida adulta, indicador de falta de suporte aos indivíduos (MEIRING et al., 2016). 
No Brasil são poucas as pesquisas que abordam a inclusão de autistas no mercado de trabalho (LEOPOLDINO, 2015; SALGADO, 2014), o que justifica a necessidade de mais investigações a respeito do assunto. As limitadas evidências existentes apontam para dificuldades compatíveis com as que foram relatadas internacionalmente, embora com especificidades próprias. A realidade brasileira apresenta peculiaridades derivadas do preconceito e desconhecimento a respeito do autismo, da pouca estrutura existente e de limitações dos sistemas de educação, saúde e justiça, aos quais muitos autistas recorrem para atendimento de direitos básicos (GOMES et al., 2015; SALGADO, 2014; NUNES; AZEVEDO; SCHMIDT, 2013).

A despeito da dificuldade em estipular o número de pessoas com autismo em nosso país (NUNES; AZEVEDO; SCHMIDT, 2013), a relevância da temática também pode ser constatada pela quantidade de pessoas com autismo. O diagnóstico precoce tem agregado mais informações sobre a prevalência do transtorno, que passou de um caso para cada 1000 pessoas na década de 1990 para um caso a cada 150 pessoas, ou menos. Dependendo do estudo, pode-se chegar a uma prevalência de mais de um caso para cada 100 pessoas (CHRISTENSEN et al., 2016; WINGATE et al., 2014; FILIPE, 2013).

Com base nesta problemática foi desenvolvido o presente ensaio teórico, construído segundo o objetivo geral: apresentar o panorama do processo inclusivo de autistas no mercado de trabalho. Como objetivos específicos evidenciam-se as seguintes metas: apresentar políticas públicas voltadas para a inclusão de autistas no mercado de trabalho; descrever o processo de inclusão de pessoas com TEA no mercado laboral; identificar obstáculos à inclusão de autistas no mercado de trabalho e, por fim, apresentar oportunidades motivadoras para a dinamização deste processo. Para subsidiar sua elaboração, foram feitas buscas na base scielo, e no google acadêmico, recuperando-se textos nacionais e estrangeiros relevantes sobre a temática abordada. A revisão de literatura foi encerrada por esgotamento, quando não foram identificadas obras relevantes, ou por saturação, para cada assunto investigado.

\section{INCLUSÃO DE AUTISTAS PARA O MERCADO DE TRABALHO}

O aumento na prevalência do TEA, que atingiu valor a cerca de $1 \%$ da população, medido em vários estudos, gera impactos substanciais na sociedade, consistindo em argumento motivador para a implementação de políticas públicas em relação ao autismo (CHRISTENSEN et al., 2016; WINGATE et al., 2014; OUELLETTE-KUNTZ et al., 2014; NEIK et al., 2014; BRUGHA et al., 2011). No Brasil, atualmente inexiste índice ou avaliação oficial para o número de pessoas com autismo e geralmente assume-se que a prevalência é similar à verificada nos estudos internacionais.

Também contribuíram para a difusão de políticas públicas fatores como a pressão dos autistas, de suas famílias e de organizações da sociedade civil e a ação de instituições internacionais (LEOPOLDINO, 2016; SHEPHERD; WADDELL, 2015; WORLD HEALTH ORGANIZATION; THE WORLD BANK, 2011). Podem ser citados como exemplos de políticas públicas inclusivas o acesso a diagnóstico precoce, a tratamentos e a educação de qualidade, adaptada às necessidades e potencialidades individuais.

Entretanto, o baixo percentual de autistas com atividade profissional e a falta de suporte para sua inclusão ensejam a aplicação de políticas específicas relativas a esta questão. Evidências apontam que intervenções bem-sucedidas podem ampliar muito as possibilidades de inclusão efetiva (WEHMAN et al., 2014), sobretudo, através de políticas públicas direcionadas a este grupo de cidadãos, o que será discutido a seguir. 


\subsection{Políticas Públicas para a Inclusão de Autistas no Mercado de Trabalho}

A adoção de políticas públicas voltadas para facilitar o ingresso de autistas no mercado de trabalho é uma tendência global, em função do elevado percentual de indivíduos com TEA excluídos em diferentes países (SEAMAN; CANNELLA-MALONE, 2016; SMITH et al., 2015; BUESCHER et al., 2014; BURGESS; CIMERA, 2014; ROUX et al., 2013; BURKE et al., 2010; HENDRICKS; WEHMAN, 2009).

Mundialmente, o grande marco para o reforço das políticas públicas foi a publicação da Convenção de Direitos das Pessoas com Deficiências em 2007 (UNITED NATIONS, 2006). Apesar de países europeus, como a Suécia e a Alemanha, adotarem desde a década de 1990 políticas públicas voltadas especificamente aos trabalhadores autistas (ROSQVIST; KEISU, 2012), a convenção, assinada inclusive pelo Brasil, abriu espaço para a formulação e aprimoramento de políticas associadas à inclusão de autistas no mercado de trabalho.

Anos após a sua assinatura, o Brasil reconheceu a necessidade de "estímulo à inserção da pessoa com transtorno do espectro autista no mercado de trabalho, observadas as peculiaridades da deficiência" através da Política Nacional de Proteção dos Direitos da Pessoa com Transtorno do Espectro Autista (BRASIL, 2012). Entretanto, não foram estabelecidos formalmente os recursos e as estruturas de serviços para a implantação das políticas, o que limitou a sua operacionalização.

Considerando-se as políticas adotadas e as necessidades das pessoas com autismo, podem ser vislumbrados quatro grandes eixos de políticas públicas relacionadas à inclusão no mercado de trabalho: Preparação para a atuação profissional; Incentivo à contratação; Fomento à produção científica; e Aquisição de informações precisas sobre a questão (LEOPOLDINO, 2015). O Quadro 1 apresenta uma descrição desses eixos/dimensões de políticas públicas e as práticas vinculadas a essas dimensões.

\section{Quadro 1 - Políticas e práticas relativas à inclusão no trabalho dos autistas}

\begin{tabular}{|c|c|}
\hline Dimensão & Práticas/ Políticas \\
\hline $\begin{array}{l}\text { Preparação para a atuação } \\
\text { profissional }\end{array}$ & $\begin{array}{l}\text { - Ensino técnico profissionalizante inclusivo } \\
\text { - Preparo vocacional individualizado } \\
\text { - Incentivos ao estágio e ao primeiro emprego } \\
\text { - Acompanhamento de assistentes sociais e psicólogos }\end{array}$ \\
\hline Incentivo à contratação & $\begin{array}{l}\text { - Incentivo financeiro a estágios e à contratação em organizações privadas } \\
\text { - Abertura de vagas de estágio e emprego em organizações públicas } \\
\text { - Programas de formação de mentores, gestores e empregadores } \\
\text { - Programas de conscientização de empregadores e gestores/ líderes } \\
\text { - Incentivo ao empreendedorismo, voluntariado e cooperativismo }\end{array}$ \\
\hline $\begin{array}{l}\text { Fomento à produção } \\
\text { científica }\end{array}$ & $\begin{array}{l}\text { - } \text { Promoção da Criação de Grupos de Pesquisas } \\
\text { - Promoção da Realização de Eventos } \\
\text { - Estímulos à Pesquisa e à Publicação } \\
\text { - }\end{array}$ \\
\hline $\begin{array}{l}\text { Aquisição de informações } \\
\text { precisas }\end{array}$ & $\begin{array}{l}\text { - Criação de cadastro nacional de autistas } \\
\text { - Censo socioeconômico dos autistas } \\
\text { - Censo de inclusão dos autistas no mercado de trabalho } \\
\text { - Ampla divulgação dos dados obtidos, com incentivo à sua pesquisa }\end{array}$ \\
\hline
\end{tabular}

Fonte: Leopoldino (2015, p. 864).

A partir do Quadro 1, é possível constatar que os quatro eixos temáticos envolvem a adoção de uma série de práticas capazes estimular a atuação profissional de autistas. Em relação à primeira dimensão, destaca-se que a preparação para a atuação profissional das pessoas com autismo envolve o acesso à educação efetiva, compreendendo ensino técnico 
profissionalizante inclusivo, preparo vocacional individualizado, incentivos ao estágio e ao primeiro emprego e o acompanhamento tanto no período de busca pelo emprego, continuando após a contratação. Pressupõe-se que os indivíduos com TEA tenham acesso prévio às instâncias de educação básica, para adquirir nível adequado de conhecimentos e funcionalidade para poderem ser preparados para o mercado, embora parte desta população não atinja este patamar em virtude da severidade do autismo (SEAMAN; CANNELLAMALONE, 2016).

A segunda dimensão tem sido utilizada em vários países como forma de tornar mais atraente a contratação de pessoas com autismo (SCOTT et al., 2017; ROSQVIST; KEISU, 2012). Essa dimensão compreende o incentivo financeiro a estágios e à contratação em organizações privadas, a abertura de vagas de estágio e emprego em organizações públicas, programas de formação de mentores, gestores e empregadores, programas de conscientização de empregadores e gestores/líderes e incentivo ao empreendedorismo, voluntariado e cooperativismo. A eficácia desta medida é aumentada caso os indivíduos tenham acesso às estruturas de preparação para a atuação profissional e apresenta potencial para ampliar o número de entidades empregadoras.

O fomento à produção científica, por sua vez, permite a exploração de questões referentes à inclusão das pessoas com TEA no mercado de trabalho, com avaliação de sua efetividade e das práticas empregadas. Compreende a promoção da criação de grupos de pesquisas, o estímulo à realização de eventos, à pesquisa, publicação e extensão universitária (LEOPOLDINO, 2015).

Por fim, a aquisição de informações precisas permite informações para a tomada de decisão relativa às políticas públicas, além de oferecer insumos informacionais sobre o autismo para a sociedade (LEOPOLDINO, 2015). A obtenção de estatísticas sobre as pessoas com TEA, a sua localização, o seu grau de funcionalidade e a sua qualidade de vida poderia suprir lacunas substanciais no conhecimento sobre o autismo. Algumas possibilidades a serem exploradas neste sentido compreendem a criação de cadastro nacional de autistas, o censo socioeconômico dos autistas, o censo de inclusão dos autistas no mercado de trabalho, além da ampla divulgação dos dados obtidos, aliada ao incentivo à sua pesquisa.

As políticas supracitadas devem ser operacionalizadas de modo a não gerar gargalos e descontinuidades. A próxima seção apresenta um processo contínuo e interativo para a inclusão laboral de pessoas com TEA.

\subsection{Processos Operacionais de Inclusão de Autistas no Mercado de Trabalho}

Em termos operacionais a inclusão de autistas no mercado de trabalho pode ser vista como processo composto por ações coordenadas de múltiplos atores que transcorre de acordo com as políticas públicas concernentes ao tema. A interação entre as atividades permite que autistas sejam inseridos e recolocados no mercado, de acordo com a necessidade. As principais atividades deste processo compreendem, conforme exposto na Figura 1: preparar os indivíduos; encaminhar esses indivíduos para o mercado; ajustar o ambiente laboral; e, acompanhar o processo. 


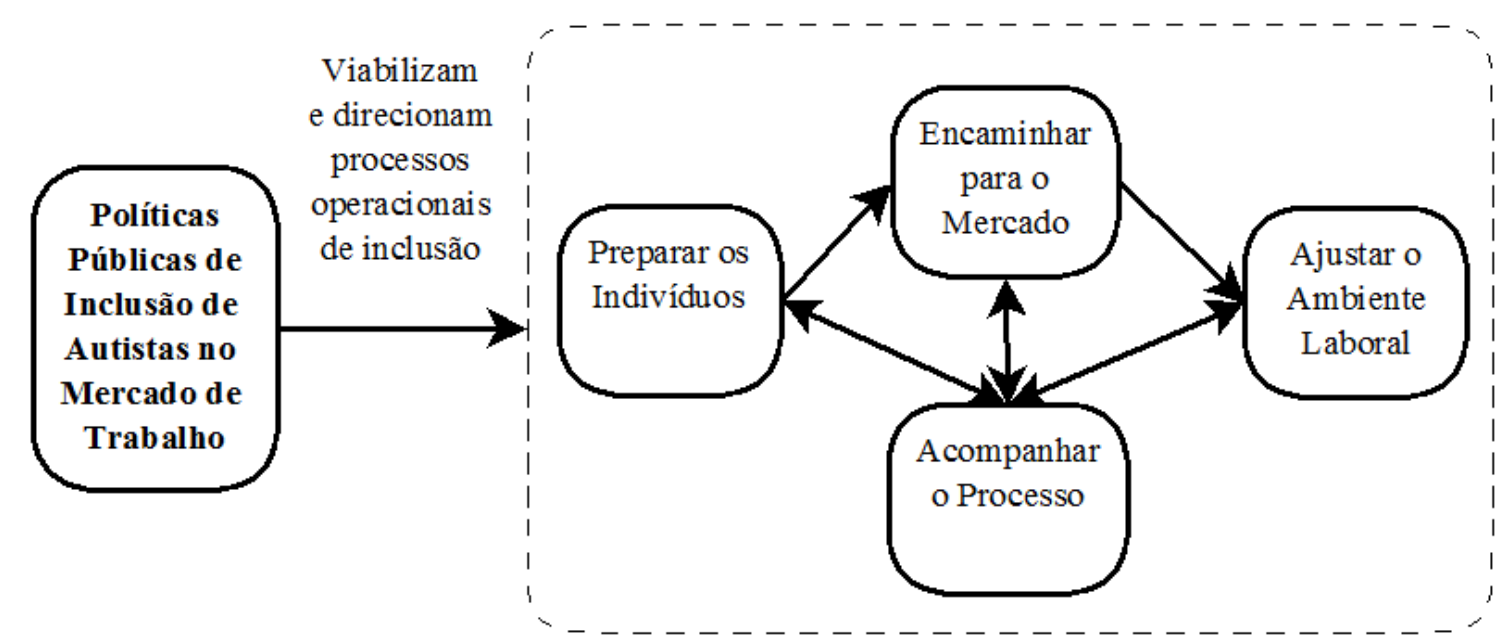

Figura 1 - Processo de inclusão de pessoas com TEA no mercado de trabalho Fonte: Elaborada pelos autores

A preparação dos indivíduos com TEA para o trabalho deve contemplar habilidades sociais, vocacionais e técnicas, permitindo maior empregabilidade e autonomia para os profissionais autistas que procuram posições no mercado de trabalho (MINATEL; MATSUKURA, 2015; GRACIOLI; BIANCHI, 2014). A inclusão prévia dos autistas no ambiente escolar é requisito importante para a obtenção de maior autonomia, facilitando a preparação para o trabalho. Adicionalmente, deve ser feita a preparação segundo os conteúdos do ensino acadêmico formal, que compõe requisitos para muitas das vagas oferecidas.

$\mathrm{O}$ encaminhamento para o mercado é essencial, pois estabelece relacionamentos entre os potenciais trabalhadores e o mercado. As entidades que realizam este serviço identificam que candidatos mais se aproximam dos requisitos que constam das vagas disponíveis pelos potenciais empregadores, e realizam o encaminhamento. Instituições públicas, privadas e não governamentais podem realizar este papel, auxiliadas por tecnologias como a de redes sociais e a dos aplicativos para dispositivos móveis. Salienta-se que mercado de trabalho pode ser ampliado através da disponibilização de vagas em instituições não governamentais e entidades comunitárias, aumentando o leque de possibilidades.

$\mathrm{O}$ ajuste do ambiente laboral para acolher os profissionais com autismo é outra necessidade a ser suprida. Este processo é responsável pela construção dos suportes material e psicossocial à inclusão. Estação de trabalho, mobiliário, iluminação e nível de ruídos são algumas das variáveis que podem ser ajustadas, assim como a comunicação e a descrição de tarefas a serem realizadas. A designação de mentores tem sido relatada como prática de suporte efetiva (HEDLEY et al., 2017; GARCÍA-VILLAMISAR; WEHMAN; NAVARRO, 2002). É importante que este processo conte com a colaboração de pessoas da entidade empregadora, envolvendo chefias e colegas de trabalho.

O acompanhamento do processo funciona como elo entre as pessoas com TEA, as entidades empregadoras, formadoras e encaminhadoras. O processo de acompanhamento permite a avaliação de eficiência e qualidade das práticas adotadas, e o seu aprimoramento contínuo. É igualmente importante mensurar a qualidade de vida dos indivíduos incluídos no processo, em suas várias dimensões, assim como sua satisfação e produtividade. Da mesma forma, faz-se necessário medir os suportes fornecidos pelas entidades empregadoras. Em paralelo ao acompanhamento, devem ser oferecidos serviços como assistência psicológica, médica e social. Caso o monitoramento encontre problemas, pode-se intervir de maneiras distintas de acordo com a necessidade, encaminhando os indivíduos para as entidades que fazem a sua preparação, direcionando-os a outra entidade empregadora, ou podem ser feitos ajustes no suporte oferecido ao trabalhador no seu ambiente laboral atual. A responsabilidade 
pelo acompanhamento pode ser compartilhada entre entes públicos, privados, não governamentais, famílias e associações de pessoas com autismo e os próprios trabalhadores com TEA.

Uma vez implementado, o processo inclusivo ajuda a mitigar os obstáculos à inserção no mercado, discutidos no tópico seguinte.

\section{OBSTÁCULOS À INCLUSÃO DE AUTISTAS NO MERCADO DE TRABALHO}

A literatura aponta diversos obstáculos à inclusão de autistas no mercado de trabalho, apresentados nesta seção. O Quadro 2 sumariza as principais restrições identificadas.

\section{Quadro 2 - Obstáculos à inclusão de autistas no mercado de trabalho}

\begin{tabular}{|c|c|c|}
\hline Obstáculo & Descrição & Referências \\
\hline $\begin{array}{l}\text { Preconceito de } \\
\text { potenciais } \\
\text { empregadores }\end{array}$ & $\begin{array}{l}\text { Percepção negativa sobre o autista, considerando a } \\
\text { contratação como uma obrigação legal que pode } \\
\text { gerar o aumento de custos. }\end{array}$ & $\begin{array}{l}\text { Robertson (2009), Wieren, Reid e } \\
\text { Mcmahon (2008) e Silva (2013) }\end{array}$ \\
\hline $\begin{array}{l}\text { Discriminação por parte } \\
\text { de colegas }\end{array}$ & $\begin{array}{l}\text { Barreira atitudinal dos demais trabalhadores em } \\
\text { integrar o autista ao ambiente de trabalho. }\end{array}$ & Orsmond et al. (2013) \\
\hline $\begin{array}{l}\text { Falta de suporte/ } \\
\text { adaptação no trabalho }\end{array}$ & $\begin{array}{l}\text { A satisfação e a produtividade do profissional } \\
\text { autista dependem da adaptação de condições } \\
\text { ambientais no trabalho - como a intensidade dos } \\
\text { ruídos - e do suporte dos colegas de trabalho. }\end{array}$ & $\begin{array}{l}\text { Parr e Hunter (2014) e Orsmond et } \\
\text { al. (2013) }\end{array}$ \\
\hline $\begin{array}{l}\text { Oferta de vagas de } \\
\text { baixa qualidade }\end{array}$ & $\begin{array}{l}\text { Remuneração dos autistas é em média menor do } \\
\text { que a de trabalhadores neurotípicos, a quantidade } \\
\text { de horas trabalhadas é menor e não há panorama } \\
\text { de crescimento profissional. }\end{array}$ & $\begin{array}{l}\text { Seaman, Cannella-Malone (2016), } \\
\text { Silva (2013), Roux et al. (2013) }\end{array}$ \\
\hline $\begin{array}{l}\text { Falta de preparo } \\
\text { vocacional }\end{array}$ & $\begin{array}{l}\text { Dificuldade em proporcionar ao adolescente } \\
\text { autista o conhecimento sobre a área em que deseja } \\
\text { atuar no mercado de trabalho. }\end{array}$ & Hillier et al. (2007) \\
\hline $\begin{array}{l}\text { Falta de Formação } \\
\text { Técnica/ Profissional }\end{array}$ & $\begin{array}{l}\text { Lacunas na formação técnica que foram originadas } \\
\text { em certos casos por falhas na inclusão prévia dos } \\
\text { indivíduos com TEA no sistema educacional. }\end{array}$ & $\begin{array}{l}\text { Gracioli e Bianchi (2014) e Toldrá } \\
\text { (2009) }\end{array}$ \\
\hline $\begin{array}{l}\text { Falta de incentivos } \\
\text { financeiros à } \\
\text { contratação }\end{array}$ & $\begin{array}{l}\text { O estímulo inicial à contratação destes } \\
\text { trabalhadores pode ser uma contrapartida } \\
\text { financeira oferecida pelo Estado à empresa. }\end{array}$ & $\begin{array}{l}\text { Scott et al (2017) e Rosqvist e } \\
\text { Keisu (2012) }\end{array}$ \\
\hline $\begin{array}{l}\text { Falta de efetividade da } \\
\text { lei de cotas }\end{array}$ & $\begin{array}{l}\text { Preferência dos potenciais empregadores em } \\
\text { contratar trabalhadores com outras deficiências }\end{array}$ & $\begin{array}{l}\text { Dos Anjos et al. (2016), Silva } \\
\text { (2013), Ribeiro e Carneiro (2009) }\end{array}$ \\
\hline
\end{tabular}

Fonte: Elaborado pelos autores

O preconceito de potenciais empregadores consiste em obstáculo substancial, pois nega totalmente o acesso a vagas em um grande número de empresas (ROSQVIST; KEISU, 2012; ROBERTSON, 2009; VAN WIEREN; REID; MCMAHON, 2008). Caracteriza-se pelo não reconhecimento da capacidade dos profissionais que apresentam autismo e pela percepção exacerbada de inconveniências e potenciais riscos incorridos na contratação de empregados com TEA. O estigma do autismo é forte a ponto de certos empregadores abertos a pessoas com deficiências diversas manterem restrições a pessoas com transtorno do espectro do autismo. A disponibilização de subsídios ao emprego de pessoas com autismo apresenta potencial para o aumento das vagas oferecidas (ROSQVIST; KEISU, 2012). 
A discriminação ou isolamento por parte de colegas ocorre de forma explícita ou implícita, restringindo a interação social substancialmente, e os laços com o trabalho dela decorrentes (ORSMOND et al., 2013; HENDRICKS, 2010). Este tipo de ocorrência é mais grave pelo fato de ser o apoio dos colegas um dos principais facilitadores na inclusão de autistas no trabalho (HEDLEY et al., 2017).

A falta de suporte ou adaptação no trabalho afeta o desempenho dos indivíduos com TEA. A atuação da liderança da instituição empregadora neste sentido pode ser determinante para o maior desempenho e atitudes mais positivas em relação ao trabalho (PARR; HUNTER, 2014). Condições ambientais como o nível de iluminação e ruídos podem ser ajustadas, assim com a organização das tarefas para a construção de rotinas adaptadas que permitam que pessoas com TEA trabalhem com produtividade e satisfação (HENDRICKS, 2010; ROBERTSON, 2009; BONTEMPO, 2009). O suporte psicossocial, oferecido pela chefia, por um coach e pelos colegas é vital para a retenção de autistas em seus empregos (PARR; HUNTER, 2014; ORSMOND et al., 2013).

A predominância de vagas de baixa qualidade desestimula o ingresso ao mercado das pessoas com deficiência. No caso das pessoas com autismo a oferta de vagas é composta em boa parte dos casos de empregos sub-remunerados, com menor carga horária e sem possibilidade de crescimento. A baixa remuneração consiste na utilização de pagamento com valor inferior ao que é oferecido no mercado, ou que não supre as necessidades e aspirações do indivíduo, e tem sido reportada em relação aos trabalhadores com TEA (SEAMAN; CANNELLA-MALONE, 2016; ROUX et al., 2013; CIMERA; BURGESS, 2011). Este é um obstáculo significativo à inclusão efetiva, pois reduz a atratividade do trabalho, piora as condições de vida e a motivação dos trabalhadores (SEAMAN; CANNELLA-MALONE, 2016; SILVA, 2013). O fato de que os autistas tendem a trabalhar menos horas também contribui para uma renda menor (ROUX et al., 2013; CIMERA; BURGESS, 2011). A falta de horizonte de crescimento ocorre em organizações que oferecem vagas para pessoas com autismo sem acreditarem em suas potencialidades. Nestes ambientes não há preocupação em oferecer oportunidades de crescimento na carreira, levando à sensação de estagnação profissional.

A falta de preparo vocacional suficiente e adequado reduz o potencial inclusivo (SEAMAN; CANNELLA-MALONE, 2016; SMITH et al., 2015). O preparo vocacional permite a reflexão sobre as necessidades e anseios individuais, a exploração de diferentes possibilidades de engajamento profissional e o melhor planejamento de carreira (SILVA, 2013), além do desenvolvimento de habilidades para aplicação prática na procura e retenção de posições no mercado de trabalho (SEAMAN; CANNELLA-MALONE, 2016). A realização de atividades neste sentido está relacionada com maior empregabilidade, mais retenção no trabalho e maiores salários (SMITH et al., 2015; HILLIER et al., 2007).

A falta de formação técnica/ profissional apresentada por muitas pessoas com autismo é um fator que restringe o acesso às profissões que exigem maior qualificação. Para se superar esta questão depende-se da inclusão prévia dos indivíduos com TEA no sistema educacional, o que está longe de ocorrer universalmente, de acordo em diversos relatos (MINATEL; MATSUKURA, 2015; GRACIOLI; BIANCHI, 2014; TOLDRÁ, 2009). No Brasil, a exigência de formação secundária e técnica para pessoas com deficiência estabelece barreiras à contratação, impedindo sua participação nos processos seletivos sem gerar passivo legal (TOLDRÁ, 2009).

A falta de incentivos financeiros à contratação de pessoas com autismo salienta-se como obstáculo importante. Tais incentivos têm sido empregados em países como Austrália e Suécia, e fazem com que a contratação de autistas seja estimulada pela disponibilização de recursos (SCOTT et al., 2017; ROSQVIST; KEISU, 2012). Ao mesmo tempo em que os 
incentivos possibilitam aos indivíduos com TEA os benefícios da inclusão, reduzem a oposição dos prováveis empregadores.

Por fim, a falta de efetividade das cotas para deficientes afeta a empregabilidade dos autistas brasileiros (ADORNO JÚNIOR; SALVATTO, 2014; TOLDRÁ, 2009). No Brasil, as cotas para deficientes foram criadas em lei promulgada em 1991. Esta lei estabelece percentuais de cotas de contratação de trabalhadores com deficiência, que variam entre 2 e 5\% de acordo com o porte da organização empregadora (BRASIL, 1991). As cotas só puderam ser utilizadas pelas pessoas com TEA após a promulgação da Política Nacional de Proteção dos Direitos da Pessoa com Transtorno do Espectro Autista, que atribuiu aos autistas os mesmos direitos previamente atribuídos aos deficientes em geral (BRASIL, 2012). No entanto, a literatura aponta a adoção intencional de atitudes visando contornar, protelar ou minimizar a contratação de cotistas com deficiência e a adoção de seletividade em favor de pessoas com menor grau de deficiência física (DOS ANJOS et al., 2016; DOS SANTOS et al., 2015; RIBEIRO; CARNEIRO, 2009; TOLDRÁ, 2009), o que exclui as pessoas com autismo do conjunto de beneficiados pela lei.

A superação de obstáculos relacionados à inclusão dos autistas no mercado laboral permite a exploração de uma série de oportunidades, atreladas inclusive ao perfil destes profissionais, e serão apresentadas a seguir.

\section{OPORTUNIDADES RELACIONADAS À INCLUSÃO DE AUTISTAS NO MERCADO DE TRABALHO}

As potencialidades manifestadas pelas pessoas com TEA que atuam no mercado de trabalho já foram constatadas em diversos estudos (AUSTIN; SONNE, 2014, SCHALL; WEHMAN; MCDONOUGH, 2012; BURKE et al., 2010; HURLBUTT; CHALMERS, 2004). De fato, as limitações na comunicação, na socialização e os comportamentos repetitivos são traços do perfil da pessoa com autismo que não constituem obstáculo insuperável, levando-se em conta as características individuais e a adequação necessária.

Alguns dos seus pontos fortes compreendem: serem amigáveis à rotina e ao cumprimento de regras; apresentarem menor taxa de atrasos e demoras nas pausas do trabalho; perderem menos tempo em conversas com colegas de trabalho ou chamadas telefônicas pessoais; poderem apresentar excelente memória para detalhes; preferirem ambientes visualmente organizados; gostar de completar tarefas; pensar de forma diferente; e, poderem apresentar habilidades e conhecimento aprofundado em áreas pelas quais tenham um interesse especial. Estas características estão descritas no Quadro 3: 
Quadro 3 - Pontos fortes do perfil do profissional com autismo

\begin{tabular}{|c|c|}
\hline $\begin{array}{l}\text { Perfil do profissional autista } \\
\text { (pontos fortes) }\end{array}$ & Descrição \\
\hline $\begin{array}{l}\text { Amigáveis à rotina e ao } \\
\text { cumprimento de regras }\end{array}$ & $\begin{array}{l}\text { Têm facilidade em trabalhar com atividades rotineiras e processos } \\
\text { padronizados, além de serem avessos ao descumprimento de normas } \\
\text { estabelecidas no ambiente de trabalho. }\end{array}$ \\
\hline $\begin{array}{l}\text { Apresentam menor taxa de atrasos e } \\
\text { demoras nas pausas do trabalho }\end{array}$ & $\begin{array}{l}\text { Em função de sua maior propensão a cumprir as regras estabelecidas para } \\
\text { realizar atividades laborais, este grupo de profissionais é pouco propenso } \\
\text { ao atraso ou a demorar nos intervalos de almoço e lanche. }\end{array}$ \\
\hline $\begin{array}{l}\text { Perdem menos tempo em conversas } \\
\text { com colegas de trabalho }\end{array}$ & $\begin{array}{l}\text { O foco para realizar as atividades e a dificuldade em interagir com as } \\
\text { demais pessoas levam estes profissionais a se dispersarem menos com } \\
\text { conversas ou chamadas telefônicas pessoais. }\end{array}$ \\
\hline $\begin{array}{l}\text { Podem apresentar excelente } \\
\text { memória para detalhes }\end{array}$ & $\begin{array}{l}\text { Possuem alta capacidade de memorizar dados e processos relativos à sua } \\
\text { atividade laboral. }\end{array}$ \\
\hline $\begin{array}{l}\text { Preferem ambientes visualmente } \\
\text { organizados }\end{array}$ & $\begin{array}{l}\text { Gostam de manter o ambiente de trabalho limpo e organizado, trazendo } \\
\text { ordem a ambientes desorganizados. }\end{array}$ \\
\hline Gostam de completar tarefas & $\begin{array}{l}\text { São profissionais que se motivam com facilidade em relação às tarefas } \\
\text { propostas e são capazes de ir além para buscar informações para completá- } \\
\text { las. }\end{array}$ \\
\hline Pensam de forma diferente & $\begin{array}{l}\text { Pensam diferentemente e podem dar respostas que fujam do pensamento } \\
\text { convencional. }\end{array}$ \\
\hline $\begin{array}{l}\text { Podem apresentar habilidades e } \\
\text { conhecimento aprofundado em } \\
\text { determinadas áreas }\end{array}$ & $\begin{array}{l}\text { Além de executar os processos com rigor, podem evidenciar um elevado } \\
\text { conhecimento sobre eles e aprimorá-los, caso possuam interesse especial } \\
\text { nas áreas em que estão atuando. }\end{array}$ \\
\hline
\end{tabular}

Além dos benefícios advindos do perfil deste profissional, a integração de autistas no mercado de trabalho apresenta oportunidades aos indivíduos incluídos, para suas famílias, aos empregadores e aos governos (HEDLEY et al., 2017; LEOPOLDINO, 2015; BUESCHER et al., 2014; ROSQVIST; KEISU, 2012; KNAPP; ROMEO; BEECHAM, 2009; ROBERTSON, 2009; GARCÍA-VILLAMISAR; HUGHES, 2007; BRASIL, 1991), como se observa no Quadro 4.

Quadro 4 - Sumário das oportunidades de inclusão

\begin{tabular}{|l|l|}
\hline \multicolumn{1}{|c|}{ Beneficiário } & \multicolumn{1}{c|}{ Oportunidades } \\
\hline \multirow{3}{*}{ Trabalhadores com TEA } & • Ganho de autonomia \\
& - Melhoria da performance cognitiva \\
& - Maior qualidade de vida do indivíduo e da família \\
\hline \multirow{2}{*}{ Empresas } & - Ganho potencial em marketing \\
& - Acesso a incentivos governamentais \\
& - Possibilidade de cumprir requisitos legais como cotas para deficientes \\
\hline \multirow{3}{*}{ Governos } & - Utilização do potencial de autistas para atividades específicas \\
\hline & - Potencial para reduzir a necessidade de gastos com assistência social para as \\
& Possibilidade de amenizar a perda de produtividade oriunda da exclusão de \\
& potenciais trabalhadores \\
\hline
\end{tabular}

Fonte: Elaborado pelos autores 
Para os trabalhadores com TEA, a inclusão no mercado de trabalho apresenta retornos em ganho de autonomia, performance cognitiva, maior qualidade de vida, melhor situação financeira e maior aplicação de aptidões desenvolvidas nas terapias e estudos realizados (HEDLEY et al., 2017; LEOPOLDINO, 2015; ROBERTSON, 2009; GARCÍAVILLAMISAR; HUGHES, 2007; GARCÍA-VILLAMISAR; WEHMAN; NAVARRO, 2002). Os ganhos supracitados refletem-se na família dos autistas, frequentemente sujeita a stress emocional e financeiro, ao preconceito e situações de isolamento social (OBEID et al., 2015; GOMES et al., 2015; MCSTAY et al., 2014; HORLIN et al., 2014; ORSMOND et al., 2013; DIVAN et al., 2012).

Para as empresas, existe o ganho potencial em marketing, acesso a incentivos governamentais, caso existam, e a possibilidade de cumprir requisitos legais como cotas para deficientes e utilizar do potencial de autistas para atividades específicas (SCOTT et al., 2017; LEOPOLDINO, 2015; AUSTIN; SONNE, 2014; ROSQVIST; KEISU, 2012; ROBERTSON, 2009; BRASIL, 1991).

No que se refere aos governos, a inclusão de autistas no mercado de trabalho apresenta potencial para reduzir a necessidade de gastos com assistência social para as pessoas com TEA e suas famílias e amenizar a perda de produtividade oriunda da exclusão de potenciais trabalhadores (BUESCHER et al., 2014; KNAPP; ROMEO; BEECHAM, 2009; GANZ, 2007). Este argumento ganha força ao se constatar que os custos associados à assistência e tratamentos de pessoas com TEA são considerados superiores aos relacionados a pessoas com outras deficiências (CIMERA; COWAN, 2009; SHIMABUKURO; GROSSE; RICE, 2008).

A literatura atesta empiricamente a relevância da inclusão de autistas no mercado de trabalho. Adicionalmente, evidências eloquentes podem ser obtidas nos textos em que os próprios indivíduos relatam seu entusiasmo e as conquistas obtidas (AYDOS, 2016). Acredita-se que o processo de inclusão de autistas apresentaria menos descontinuidades e mais eficiência com o aumento da participação dos envolvidos no processo, o que compreende também atores do universo empresarial. Desta forma, as oportunidades identificadas poderiam ser oferecidas mais amplamente e ser mais bem aproveitadas pelos interessados.

\section{CONCLUSÃO}

Discussões relativas à inclusão de pessoas com autismo no mercado de trabalho são escassas, principalmente na área de Administração. A despeito da representatividade deste grupo de cidadãos e dos recentes avanços legais, a maioria das organizações brasileiras ainda não oferece condições mínimas para a inclusão de profissionais autistas em seus quadros de funcionários. Em função disso, este estudo objetivou apresentar o panorama do processo inclusivo de autistas no mercado de trabalho, através do debate de políticas públicas voltadas para a sua inclusão e do processo de inclusão de pessoas com TEA, além dos obstáculos e oportunidades para o processo inclusivo. A partir da problematização apresentada nos tópicos anteriores, baseada primordialmente em estudos estrangeiros da área da Saúde e Educação, alguns pontos merecem ser destacados.

Primeiramente, os recentes avanços na legislação brasileira voltados à inclusão de pessoas com transtorno do espectro autista não foram acompanhados de recursos e estruturas para a sua implantação. Dessa forma, o Estado ainda não parece capaz de mitigar a exclusão destes profissionais no mercado de trabalho. As barreiras em relação ao ambiente laboral podem ser amenizadas com a atuação dos agentes públicos em quatro eixos principais: 
preparação para a atuação profissional; incentivo à contratação; fomento à produção científica; e aquisição de informações precisas sobre a questão.

Em relação ao processo inclusivo, é necessário destacar que o mesmo é operacionalizado por diferentes indivíduos e organizações, podendo ser particionado em quatro grandes atividades: preparação dos indivíduos; encaminhamento para o mercado; ajuste do ambiente laboral; e acompanhamento do processo. Este percurso apresenta dificuldades para a sua consecução. Parte dos entraves é manifestada pelos empregadores, como a falta de suporte no ambiente de trabalho e a oferta de vagas de baixa qualidade. Outras restrições resultam da falta de preparo vocacional das pessoas com autismo e da ineficácia das cotas para deficientes.

Esses entraves, contudo, podem ser amenizados com a exploração das potencialidades das pessoas com TEA, como a facilidade em executar funções repetitivas e memorizar detalhes, além do foco que possuem para completar tarefas e cumprirem regras estabelecidas no ambiente de trabalho. Além dos benefícios advindos do perfil deste profissional, a integração de autistas no mercado de trabalho apresenta oportunidades para os indivíduos incluídos, os empregadores e o Estado.

No que diz respeito à contribuição acadêmica deste estudo, ressalta-se que, diferentemente da maioria dos trabalhos brasileiros anteriores sobre a temática, que observam a inclusão de pessoas com autismo sob o olhar dos profissionais de saúde e educação, esta pesquisa amplia a discussão sobre o assunto sob a perspectiva da Administração, abordando os desafios e potencialidades da inclusão para gestores públicos e privados.

Os resultados apresentados oferecem, sobretudo, insights para o desenvolvimento de futuros estudos empíricos sobre o assunto. A eficácia de políticas públicas voltadas para pessoas com TEA e os desafios para a inclusão são lacunas de pesquisa que podem ser exploradas em contextos nacionais diversos, visto que estudos sobre a temática em Administração são escassos.

\section{REFERÊNCIAS}

ADORNO JÚNIOR, H. L.; SALVATTO, M. V. A inclusão das pessoas com deficiência no mercado de trabalho brasileiro. UNIVERSITAS, n. 13, 2014.

AUSTIN, R. D.; SONNE, T. The Dandelion Principle: Redesigning work for the innovation economy. MIT Sloan Management Review, v. 55, n. 4, p. 67, 2014.

AYDOS, V. Agência e subjetivação na gestão de pessoas com deficiência: a inclusão no mercado de trabalho de um jovem diagnosticado com autismo. Horizontes Antropológicos, n. 46, p. $329-358,2016$.

BONTEMPO, T. Sensory Processing Patterns in High-Ability Adults with Autism Spectrum Disorders in the Workplace. Dissertação (Mestrado em Ciência da Reabilitação) - Kingston, Ontario, Queen's College, 2009.

BRASIL. LEI N ${ }^{0}$ 8.213. 1991. Dispõe sobre os Planos de Benefícios da Previdência Social e dá outras providências. Brasília: Presidência da República, 1991.

BRASIL. Lei 12.764/2012. Institui a Política Nacional de Proteção dos Direitos da Pessoa com Transtorno do Espectro Autista. Brasília: Presidência da República, 2012. 
BRUGHA, T. S. et al. Epidemiology of autism spectrum disorders in adults in the community in England. Archives of general psychiatry, v. 68, n. 5, p. 459-465, 2011.

BUESCHER, A. V. S. et al. Costs of autism spectrum disorders in the United Kingdom and the United States. JAMA pediatrics, v. 168, n. 8, p. 721-728, 2014.

BURGESS, S.; CIMERA, R. E. Employment outcomes of transition-aged adults with autism spectrum disorders: A state of the states report. American journal on intellectual and developmental disabilities, v. 119, n. 1, p. 64-83, 2014.

BURKE, R. V. et al. Evaluation of two instruction methods to increase employment options for young adults with autism spectrum disorders. Research in developmental disabilities, $\mathrm{v}$. 31, n. 6, p. 1223-1233, 2010.

CHRISTENSEN, D. L. et al. Prevalence and characteristics of autism spectrum disorder among 4-year-old children in the autism and developmental disabilities monitoring network. Journal of Developmental \& Behavioral Pediatrics, v. 37, n. 1, p. 1-8, 2016.

CIMERA, R. E.; COWAN, R. J. The costs of services and employment outcomes achieved by adults with autism in the US. Autism, v. 13, n. 3, p. 285-302, 2009.

CIMERA, R. E.; BURGESS, S. Do adults with autism benefit monetarily from working in their communities?. Journal of Vocational Rehabilitation, v. 34, n. 3, p. 173-180, 2011.

DAVIDSON, C. Management of autism in France: "a huge job to be done". The Lancet Psychiatry, v. 1, n. 2, p. 113-114, 2014.

DIVAN, G. et al. Challenges, coping strategies, and unmet needs of families with a child with autism spectrum disorder in Goa, India. Autism Research, v. 5, n. 3, p. 190-200, 2012.

DOS ANJOS, M. C. G. et al. A Pessoa com Deficiência no Mercado de Trabalho e suas Implicações. Id on Line Revista de Psicologia, v. 10, n. 29, p. 51-70, 2016.

DOS SANTOS, G. C. et al. DESCORTINANDO UMA REALIDADE: PESSOAS COM DEFICIÊNCIA NAS EMPRESAS DE PASSO FUNDO/RS. Revista Cadernos de Ciências Sociais da UFRPE, v. 1, n. 2, p. 62-83, 2015.

FILIPE, T. M. G. As especificidades da orientação profisssional em clientes com perturbações do espectro do autismo/síndrome de Asperger. 2013. 195f. Dissertação (Mestrado em Psicologia) - Lisboa, UL - Universidade de Lisboa.

GANZ, M. L. The lifetime distribution of the incremental societal costs of autism. Archives of pediatrics \& adolescent medicine, v. 161, n. 4, p. 343-349, 2007.

GARCÍA-VILLAMISAR, D.; WEHMAN, P.; NAVARRO, M. D. Changes in the quality of autistic people's life that work in supported and sheltered employment. A 5-year follow-up study. Journal of Vocational Rehabilitation, v. 17, n. 4, p. 309-312, 2002. 
GARCÍA-VILLAMISAR, D.; HUGHES, C. Supported employment improves cognitive performance in adults with autism. Journal of intellectual disability research, v. 51, n. 2, p. 142-150, 2007.

GOMES, P. et al. Autism in Brazil: a systematic review of family challenges and coping strategies. Jornal de pediatria, v. 91, n. 2, p. 111-121, 2015.

GRACIOLI, M. M.; BIANCHI, R. C. Educação do autista no ensino regular: um desafio à prática pedagógica. Nucleus, v. 11, n. 2, p. 125-138, 2014.

HARMON, A. Autistic and seeking a place in an adult world. New York Times, p. A1, 2011.

HEDLEY, D. et al. Transition to work: perspectives from the autism spectrum. Autism, p. 114, 2017.

HENDRICKS, D. Employment and adults with autism spectrum disorders: challenges and strategies for success. Journal of Vocational Rehabilitation, v. 32, n. 2, p. 125-134, 2010.

HENDRICKS, D. R.; WEHMAN, P. Transition from school to adulthood for youth with autism spectrum disorders: review and recommendations. Focus on Autism and Other Developmental Disabilities, 2009.

HILLIER, A. et al. Two-year evaluation of a vocational support program for adults on the autism spectrum. Career Development for Exceptional Individuals, v. 30, n. 1, p. 35-47, 2007.

HORLIN, C. et al. The cost of autism spectrum disorders. PloS one, v. 9, n. 9, 2014.

HURLBUTT, K; CHALMERS, L. Employment and adults with Asperger syndrome. Focus on autism and other developmental disabilities, v. 19, n. 4, p. 215-222, 2004.

KLIN, A. Autismo e síndrome de Asperger: uma visão geral. Revista Brasileira de Psiquiatria. São Paulo, v. 28, suppl.1, p.s3-s11, 2006.

KNAPP, M.; ROMEO, R.; BEECHAM, J. Economic cost of autism in the UK. Autism, v. 13, n. 3, p. 317-336, 2009.

LEOPOLDINO, C. B. Inclusão de autistas no mercado de trabalho: uma nova questão de pesquisa para os brasileiros. Gestão e Sociedade, v. 9, n. 22, p. 853-868, 2015.

LEOPOLDINO, C. B. Associativismo em famílias de autistas: a gênese da Associação Fortaleza Azul. Revista Contribuciones a las Ciencias Sociales. Abr-jun. 2016.

MCSTAY, R. L. et al. Parenting stress and autism: the role of age, autism severity, quality of life and problem behaviour of children and adolescents with autism. Autism, v. 18, n. 5, p. 502-510, 2014.

MEIRING, M. et al. Transition for adolescents with autism spectrum disorder: South African parent and professional perspectives. Frontiers in psychiatry, v. 7, 2016. 
MINATEL, M. M.; MATSUKURA, T. S. Familiares de crianças e adolescentes com autismo: percepções do contexto escolar. Revista Educação Especial, v. 28, n. 52, p. 429-442, 2015.

NEIK, T. T. X. et al. Prevalence, diagnosis, treatment and research on autism spectrum disorders (ASD) in Singapore and Malaysia. International Journal of Special Education, v. 29, n. 3, p. 82-92, 2014.

NUNES, D. R. de P.; AZEVEDO, M. Q. O. de; SCHMIDT, C. Inclusão educacional de pessoas com autismo no Brasil: uma revisão da literatura. Revista Educação Especial, v. 26, n. 47, p. $557-572,2013$.

OBEID, R. et al. A cross-cultural comparison of knowledge and stigma associated with autism spectrum disorder among college students in Lebanon and the United States. Journal of autism and developmental disorders, v. 45, n. 11, p. 3520-3536, 2015.

ORSMOND, G. I. et al. Social participation among young adults with an autism spectrum disorder. Journal of autism and developmental disorders, v. 43, n. 11, p. 2710-2719, 2013.

OUELLETTE-KUNTZ, $\mathrm{H}$. et al. The changing prevalence of autism in three regions of Canada. Journal of autism and developmental disorders, v. 44, n. 1, p. 120-136, 2014.

PARR, A. D.; HUNTER, S. T. Enhancing work outcomes of employees with autism spectrum disorder through leadership: Leadership for employees with autism spectrum disorder.

Autism, v. 18, n. 5, p. 545-554, 2014.

RIBEIRO, M. A.; CARNEIRO, R. A inclusão indesejada: as empresas brasileiras face à lei de cotas para pessoas com deficiência no mercado de trabalho. Organizações e Sociedade, v. 16 , n. 50, 2009.

ROBERTSON, S. M. Neurodiversity, quality of life, and autistic adults: shifting research and professional focuses onto real-life challenges. Disability Studies Quarterly, v. 30, n. 1, 2009.

ROSQVIST, H. B.; KEISU, B. Adaptation or recognition of the autistic subject? Reimagining autistic work life: deconstructing the notion of "real jobs" in the swedish autistic selfadvocacy movement. Journal of Vocational Rehabilitation, v. 37, n. 3, p. 203-212, 2012.

ROUX, A. M. et al. Postsecondary employment experiences among young adults with an autism spectrum disorder. Journal of the American Academy of Child \& Adolescent Psychiatry, v. 52, n. 9, p. 931-939, 2013.

SALGADO, A. C. L. A inserção de autistas no mercado de trabalho brasileiro. Alethes, v. 4, n. 6, p. 421-438, jul./dez, 2014.

SCHALL, C.; WEHMAN, P.; MCDONOUGH, J. L. Transition from school to work for students with autism spectrum disorders: understanding the process and achieving better outcomes. Pediatric Clinics of North America, v. 59, n. 1, p. 189-202, 2012. 
SCOTT, M. et al. Employers' perception of the costs and the benefits of hiring individuals with autism spectrum disorder in open employment in Australia. PloS one, v. 12, n. 5, p. e0177607, 2017.

SILVA, A. C. M. da. Autismo: o acesso ao trabalho como efetivação dos direitos humanos. 2013. 203 f. Dissertação (Mestrado em Direito) - Recife, UNICAP - Universidade Católica de Pernambuco, Recife.

SEAMAN, R. L.; CANNELLA-MALONE, H. I. Vocational skills interventions for adults with autism spectrum disorder: a review of the literature. Journal of Developmental and Physical Disabilities, v. 28, n. 3, p. 479-494, 2016.

SHEPHERD, C. A.; WADDELL, C. A qualitative study of autism policy in Canada: seeking consensus on children's services. Journal of autism and developmental disorders, v. 45, n. 11 , p. 3550-3564, 2015.

SHIMABUKURO, T. T.; GROSSE, S. D.; RICE, C. Medical expenditures for children with an autism spectrum disorder in a privately insured population. Journal of autism and developmental disorders, v. 38, n. 3, p. 546-552, 2008.

SMITH, M, J. et al. Brief report: Vocational outcomes for young adults with autism spectrum disorders at six months after virtual reality job interview training. Journal of autism and developmental disorders, v. 45, n. 10, p. 3364-3369, 2015.

TEIXEIRA, G. Manual do autismo. Rio de Janeiro: Best Seller, 2016.

TOLDRÁ, R. C. Políticas afirmativas: opinião das pessoas com deficiência acerca da legislação de reserva de vagas no mercado de trabalho. Revista de Terapia Ocupacional da Universidade de São Paulo, v. 20, n. 2, p. 110-117, 2009.

UNITED NATIONS. Convenção sobre os direitos das pessoas com deficiência da ONU. 2006.

VAN WIEREN, T. A.; REID, C. A.; MCMAHON, B. T. Workplace discrimination and autism spectrum disorders: the national EEOC americans with disabilities act research project. Work, v. 31, n. 3, p. 299-308, 2008.

WEHMAN, P. H. et al. Competitive employment for youth with autism spectrum disorders: Early results from a randomized clinical trial. Journal of autism and developmental disorders, v. 44, n. 3, p. 487-500, 2014.

WINGATE, M. et al. Prevalence of autism spectrum disorder among children aged 8 yearsautism and developmental disabilities monitoring network, 11 sites, United States, 2010. MMWR Surveillance Summaries, v. 63, n. 2, 2014.

WORLD HEALTH ORGANIZATION; THE WORLD BANK. World report on disability. World Health Organization and The World Bank, 2011. 